\title{
Mixed-Valence Lanthanide-Activated Phosphors: Invariance of the Intervalence Charge Transfer (IVCT) Absorption Onset across the Series
}

\author{
Ivo Neefjes ${ }^{1}$, Jonas J. Joos², Zoila Barandiarán ${ }^{3}$, Luis Seijo ${ }^{3}$ \\ ${ }^{1}$ Department of Chemistry, KU Leuven, Celestijnenlaan 200F, 3001 Leuven, Belgium \\ ${ }^{2}$ LumiLab, Department of Solid State Sciences, Ghent University, Krijgslaan 281-S1, 9000 Gent, Belgium. \\ Center for Nano- and Biophotonics (NB Photonics), Ghent University, Belgium \\ ${ }^{3}$ Departamento de Química, Instituto Universitario de Ciencia de Materiales Nicolás Cabrera, and Condensed \\ Matter Physics Center (IFIMAC), Universidad Autónoma de Madrid, 28049 Madrid, Spain \\ E-mail: jonas.joos@ugent.be
}

This paper was published in J. Phys. Chem. C (Available online January 6, 2020)

This version is the unedited, accepted version after peer review.

The edited version can be found at:

https://pubs.acs.org/doi/abs/10.1021/acs.jpcc.9b11084

THE JOURNAL OF

PHYSICAL CHEMISTRY

pubs.acs.org/JPCC

Mixed-Valence Lanthanide-Activated Phosphors: Invariance of the Intervalence Charge Transfer (IVCT) Absorption Onset across the Series

Ivo Neefjes, Jonas J. Joos, Zoila Barandiarán, and Luis Seijo* 


\title{
Mixed-Valence Lanthanide-Activated Phosphors: Invariance of the Intervalence Charge Transfer (IVCT) Absorption Onset Across the Series.
}

\author{
Ivo Neefjes, ${ }^{\dagger}$ Jonas J. Joos, ${ }^{\ddagger}$ Zoila Barandiarán, ${ }^{\Uparrow}$ and Luis Seijo*, \\ $\dagger$ Department of Chemistry, KU Leuven, Celestijnenlaan 200F, B-3001 Leuven, Belgium \\ $\ddagger$ LumiLab, Department of Solid State Sciences, and Center for Nano- and Biophotonics \\ (NB-Photonics), Ghent University, B-9000 Ghent, Belgium \\ \Departamento de Química, Instituto Universitario de Ciencia de Materiales Nicolás \\ Cabrera, and Condensed Matter Physics Center (IFIMAC), Universidad Autónoma de \\ Madrid, 28049 Madrid, Spain \\ E-mail: luis.seijo@uam.es
}

\section{Abstract}

Recently, direct evidence was given for the existence of intervalence charge transfer (IVCT) states of $\mathrm{Eu}^{2+} / \mathrm{Eu}^{3+}$ pairs in Eu-doped fluorite hosts and their signature in absorption spectra was characterized [J. Phys. Chem. Lett. 10(2019)1851]. Here we show, by means of multiconfigurational ab initio calculations, that the IVCT absorptions of all $2+/ 3+$ mixed valence lanthanides doped in $\mathrm{CaF}_{2}$ start basically at the same energy. This is rationalized with a simplified model of IVCT absorptions. Emissions above that energy are expected to be partly or totally quenched when $2+/ 3+$ pairs are formed. On the basis of this finding and existing calculations, we report expected IVCT absorptions of mixed valence lanthanides doped in several fluoride, oxide, and sulfide hosts.

\section{Graphical TOC Entry}

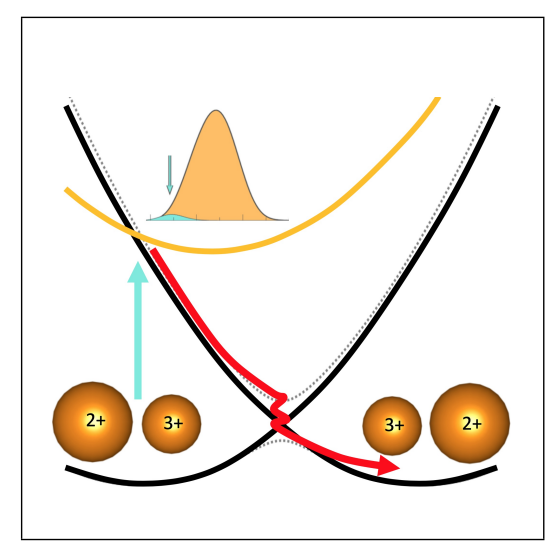

\section{Keywords}

Intervalence charge transfer, IVCT, Europium, $\mathrm{Eu}, \mathrm{CaF}_{2}$, fluorite, luminescence quenching 


\section{Introduction}

According to IUPAC recommendations, intervalence charge transfer (IVCT) is the name given to an electron transfer between two metal sites differing only in oxidation state, $\mathrm{M}^{n+}-\mathrm{M}^{(n+1)+} \rightarrow \mathrm{M}^{(n+1)+}-\mathrm{M}^{n+}, 1,2$ even though it is sometimes used for electron transfer between different metals. IVCT states have an important potential to impact the luminescence of lanthanide activated inorganic phosphors by quenching and excitation loss. Yet, they are very elusive for direct experimental measurements and not much is known about them (their absorption bands are extremely broad and flat, their intensity is low, and no emission follows the IVCT absorptions). ${ }^{3}$ This is why we dedicate this paper to studying the energies of IVCT states of mixed valence lanthanide ions doped in crystalline solids. We focus on the onsets of optical IVCT absorptions across the lanthanide series, which we study by means of state-of-theart multiconfigurational $a b$ initio calculations. We discuss next the antecedents.

Whereas the literature on IVCT between transition metal ions is abundant, ${ }^{4-6}$ the number of studies on IVCT between lanthanide ions is very scarce. Among the latter, the majority focus on thermally induced electron transfer in mixed valence compounds, the pioneering work of Berkooz et al. ${ }^{7}$ in the mixed valence Europium sulfide $\mathrm{Eu}_{3} \mathrm{~S}_{4}$ being a good example. However, very few pay attention to photoinduced electron transfer, which should provide the information on the IVCT state energies and their potential impact on luminescence. The reports by Wickleder on $\mathrm{Eu}^{2+} / \mathrm{Eu}^{3+}$ IVCT absorption bands in the mixed valence Europium chlorides $\mathrm{Na}_{5} \mathrm{Eu}_{7} \mathrm{Cl}_{22}$ and $\mathrm{KEu}_{2} \mathrm{Cl}_{6}{ }^{8,9}$ are an exception to this.

In lanthanide-doped crystalline materials the number of studies on IVCT states has been very limited. This is so in spite of the fact that these lanthanide-activated materials play a crucial role as inorganic phosphors with many technological applications, ranging from light-emitting diodes, scintillators or radiation dosimeters, ${ }^{10-13}$ to glow-in-the-dark road marks, thermometry or infrared-phosphor- based in-vivo medical imaging or theranostics. ${ }^{14-16}$ As a matter of fact, for decades, only the tentative assignment of a broad band in the reflection spectrum of Ce-doped $\mathrm{LaPO}_{4}$ as a $\mathrm{Ce}^{3+} / \mathrm{Ce}^{4+}$ IVCT absorption by van Schaik et al. ${ }^{17}$ could be cited. Recently, the importance of these materials as phosphors and the suspicion that the presence of mixed valence dopant pairs or clusters might have an important impact on their luminescence, boosted new research on the role of electron transfer between two oxidation states of the optically active dopants. E.g., the anomalous emissions of Ce-doped elpasolites and Yb-doped fluorides, and the laser-induced white emission from Ce in $\mathrm{Sr}_{2} \mathrm{CeO}_{4}$, have been interpreted as IVCT luminescences with the assistance of multiconfigurational ab initio calculations. ${ }^{18-20} \mathrm{Di}-$ rect experimental proof of the IVCT nature of excited states is still a big challenge, but recent experiments showed evidence that the anomalous emission of $\mathrm{CaF}_{2}: \mathrm{Yb}^{2+}$ is not due to impurity-trapped-excitons, ${ }^{21}$ which was the accepted model during three decades. They also demonstrated the true $\mathrm{Yb}^{2+} / \mathrm{Yb}^{3+}$ mixed valence nature of Ytterbium in $\mathrm{CaF}_{2}: \mathrm{Yb}^{2+}$ and its inherent complexity, ${ }^{22}$ so moving the focus towards the importance of IVCT states.

In spite of the elusiveness of the IVCT states, direct evidence of IVCT absorptions of $\mathrm{Eu}^{2+} / \mathrm{Eu}^{3+}$ pairs in Eu-doped $\mathrm{CaF}_{2}, \mathrm{SrF}_{2}$, and $\mathrm{BaF}_{2}$ was recently provided in a combined experimental and $a b$ initio study. ${ }^{3}$ The compatibility of the IVCT states with $\mathrm{Eu}^{2+} d-f$ luminescence in $\mathrm{CaF}_{2}$ and $\mathrm{SrF}_{2}$ was shown to turn into IVCT-driven $d-f$ luminescence quenching in $\mathrm{BaF}_{2},{ }^{3}$ which was a clear example of the potential impact of IVCT states in phosphors.

Here, we report multiconfigurational $a b$ initio calculations of the IVCT absorptions of $\mathrm{Ln}^{2+} / \mathrm{Ln}^{3+}$ pairs in Ln-doped $\mathrm{CaF}_{2}$ for the entire lanthanide series $(\mathrm{Ln}=\mathrm{Ce}, \mathrm{Pr}, \mathrm{Nd}, \mathrm{Pm}, \mathrm{Sm}$, $\mathrm{Eu}, \mathrm{Gd}, \mathrm{Tb}, \mathrm{Dy}, \mathrm{Ho}, \mathrm{Er}, \mathrm{Tm}, \mathrm{Yb})$. They are useful, e.g., for understanding the potential role of divalent/trivalent lanthanide ions when present -on purpose or unintentionallyin hosts activated with the trivalent/divalent counterparts. This is interesting because, although only a few divalent doped lanthanide 
ions are stable in regular conditions, most of them have been stabilized under suitable treatments, like x-ray and gamma irradiation ${ }^{23-25}$ and, as a matter of fact, the entire series of rare earths can exist in the divalent state when present in small amounts in crystalline $\mathrm{CaF}_{2} \cdot{ }^{23}$

We show that the computed IVCT states of all $\mathrm{Ln}^{2+} / \mathrm{Ln}^{3+}$ lanthanide pairs in $\mathrm{CaF}_{2}$ start at basically the same energy. This finding is compatible with a simple model of IVCT states, ${ }^{26}$ according to which, for given ligands and coordination number, the maximum of the absorption to the first IVCT state (i.e. the onset of the full IVCT absorption band) depends only on two simple structural properties of the electron donor and acceptor active centers: the difference between their lanthanide-ligand bond lengths, $d\left(\mathrm{Ln}^{2+}-\mathrm{L}\right)-d\left(\mathrm{Ln}^{3+}-\mathrm{L}\right)$, and the average of their breathing mode vibrational frequencies. Based on this result, we report predicted IVCT absorptions of lanthanide ions in a few other hosts where $a b$ initio calculations of these properties are available.

The paper opens the door to predicting IVCT states onsets of any lanthanide mixed valence pair in a host with relatively simple structural calculations of two oxidation states of a single lanthanide element in the host. Other alternatives for the prediction of the onsets are experimental measurements (on one lanthanide element only) of vibrational frequencies and bond length differences or their empirical estimates (e.g. using ionic radii for the latter ${ }^{26}$ ).

\section{Theoretical methods}

We computed the diabatic potential energy surfaces for electron transfer states of $\mathrm{Ln}^{2+} / \mathrm{Ln}^{3+}$ mixed valence pairs in $\mathrm{CaF}_{2}(\mathrm{Ln}=\mathrm{Ce}, \mathrm{Pr}, \mathrm{Nd}$, Pm, Sm, Eu, Gd, Tb, Dy, Ho, Er, Tm, Yb), and the corresponding IVCT configuration coordinate energy level diagrams, using the results of independent embedded cluster calculations as proposed in Refs. 18 and 19.

We assumed the lanthanide ions substitute for $\mathrm{Ca}^{2+}$ in $\mathrm{CaF}_{2}$ and we calculated the electronic structures of the electron donor and acceptor octahedral embedded clusters $\left(\mathrm{LnF}_{8}\right)^{6-}$
Table 1: Lowest free-ion and $O_{h}$ terms.

\begin{tabular}{|c|c|c|c|c|}
\hline $\operatorname{Ln}^{2+}$ & $\mathrm{Ln}^{3+}$ & Config. & $\begin{array}{c}\text { Free-ion } \\
\text { term }\end{array}$ & $O_{h}$ terms \\
\hline & $\mathrm{Ce}^{3+}$ & $4 f^{1}$ & ${ }^{2} F^{o}$ & ${ }^{2} A_{2 u},{ }^{2} T_{1 u},{ }^{2} T_{2 u}$ \\
\hline $\mathrm{Ce}^{2+}$ & $\operatorname{Pr}^{3+}$ & $4 f^{2}$ & ${ }^{3} H$ & ${ }^{3} E_{g}, 2{ }^{3} T_{1 g},{ }^{3} T_{2 g}$ \\
\hline $\operatorname{Pr}^{2+}$ & $\mathrm{Nd}^{3+}$ & $4 f^{3}$ & ${ }^{4} I^{o}$ & ${ }^{4} A_{1 u},{ }^{4} A_{2 u},{ }^{4} E_{u},{ }^{4} T_{1 u}, 2{ }^{4} T_{2 u}$ \\
\hline $\mathrm{Nd}^{2+}$ & $\mathrm{Pm}^{3+}$ & $4 f^{4}$ & ${ }^{5} I$ & ${ }^{5} A_{1 g},{ }^{5} A_{2 q},{ }^{5} E_{g},{ }^{5} T_{1 g}, 2{ }^{5} T_{2 g}$ \\
\hline $\mathrm{Pm}^{2+}$ & $\mathrm{Sm}^{3+}$ & $4 f^{5}$ & ${ }^{6} \mathrm{H}^{\circ}$ & ${ }^{6} E_{u}, 2{ }^{6} T_{1 u},{ }^{6} T_{2 u}$ \\
\hline $\mathrm{Sm}^{2+}$ & $\mathrm{Eu}^{3+}$ & $4 f^{6}$ & ${ }^{7} \mathrm{~F}$ & ${ }^{7} A_{2 g},{ }^{7} T_{1 g},{ }^{7} T_{2 g}$ \\
\hline $\mathrm{Eu}^{2+}$ & $\mathrm{Gd}^{3+}$ & $4 f^{7}$ & ${ }^{8} S^{\circ}$ & ${ }^{8} A_{1 u}$ \\
\hline $\mathrm{Gd}^{2+}$ & $\mathrm{Tb}^{3+}$ & $4 f^{8}$ & ${ }^{7} \mathrm{~F}$ & ${ }^{7} A_{2 g},{ }^{7} T_{1 g},{ }^{7} T_{2 g}$ \\
\hline $\mathrm{Tb}^{2+}$ & $\mathrm{Dy}^{3+}$ & $4 f^{9}$ & ${ }^{6} \mathrm{H}^{\circ}$ & ${ }^{6} E_{u}, 2{ }^{6} T_{1 u},{ }^{6} T_{2 u}$ \\
\hline $\mathrm{Dy}^{2+}$ & $\mathrm{Ho}^{3+}$ & $4 f^{10}$ & ${ }^{5} I$ & ${ }^{5} A_{1 g},{ }^{5} A_{2 q},{ }^{5} E_{g},{ }^{5} T_{1 g}, 2^{5} T_{2 g}$ \\
\hline $\mathrm{Ho}^{2+}$ & $\mathrm{Er}^{3+}$ & $4 f^{11}$ & ${ }^{4} I^{o}$ & ${ }^{4} A_{1 u},{ }^{4} A_{2 u},{ }^{4} E_{u},{ }^{4} T_{1 u}, 2{ }^{4} T_{2 u}$ \\
\hline $\mathrm{Er}^{2+}$ & $\mathrm{Tm}^{3+}$ & $4 f^{12}$ & ${ }^{3} \mathrm{H}$ & ${ }^{3} E_{g}, 2{ }^{3} T_{1 g},{ }^{3} T_{2 g}$ \\
\hline $\mathrm{Tm}^{2+}$ & $\mathrm{Yb}^{3+}$ & $4 f^{13}$ & ${ }^{2} F^{o}$ & ${ }^{2} A_{2 u},{ }^{2} T_{1 u},{ }^{2} T_{2 u}$ \\
\hline $\mathrm{Yb}^{2+}$ & & $4 f^{14}$ & ${ }^{1} S$ & ${ }^{1} A_{1 g}$ \\
\hline
\end{tabular}

and $\left(\mathrm{LnF}_{8}\right)^{5-}$. We also assumed a non-local long-range charge compensation for $\mathrm{Ln}^{3+}$. We performed the calculations with the suite of programs MOLCAS, ${ }^{27}$ using $D_{2 h}$ symmetry, in two step spin-orbit coupling SA-CASSCF/MSCASPT2/RASSI-SO DKH calculations that are described next: In a first step, we used the spin-orbit-free many-electron relativistic second-order Douglas-Kroll-Hess (DKH) Hamiltonian $^{28,29}$ and performed all-electron calculations with the same type of basis set used in the calculations of $\mathrm{CaF}_{2}: \mathrm{Yb}^{2+}, \mathrm{Yb}^{3+}$ in Ref. 19: Gaussian atomic natural orbital relativistic basis sets ANO-RCC for the lanthanides ${ }^{30}$ and Fluorine, ${ }^{31}$ with respective contractions $(25 s 22 p 15 d 11 f 4 g 2 h) /[9 s 8 p 5 d 4 f 3 g 2 h]$ (quadruple-zeta with polarization quality) and $(14 s 9 p 4 d) /[5 s 4 p 3 d]$ (quadruple-zeta with polarization without $f$-functions quality); plus the occupied $3 s$ and $3 p$ orbitals of the embedding $\mathrm{Ca}^{2+}$ ions, contracted as $(20 s 15 p) /[1 s 1 p]$ and located at the 12 neighbour sites $\left(\frac{1}{2}, \frac{1}{2}, 0\right)$, which act as cluster-host orthogonalization functions; ${ }^{32}$ and 5 s-type Gaussian type functions located at the $\operatorname{six}\left(\frac{1}{2}, 0,0\right)$ interstitial sites surrounding the $\mathrm{LnF}_{8}$ cube in the fluorite structure, whose orbital exponents were optimized to give minimal impurity-trapped exciton energy in $\mathrm{CaF}_{2}: \mathrm{Yb}^{2+}{ }^{19}$ In this step, we performed state-average restricted-activespace self-consistent-field (SA-CASSCF) ${ }^{33-35}$ calculations allowing all possible occupations in the Ln $4 f$ shells and up to 4 electrons in 
the Ln $5 f$ shells, in order to account for the so-called double-shell effect, whose importance when dealing with orbital shells of different occupancies was originally shown in transition metal ions ${ }^{36,37}$ and more recently confirmed in lanthanide ions. ${ }^{38}$ In these SA-CASSCF calculations, we averaged all states of equal spin and spatial irreducible representation that result from the ligand-field splitting of the lowest free-ion lanthanide ion terms; they are listed in Table 1. Subsequent multi-state secondorder perturbation theory (MS-CASPT2) ${ }^{39-42}$ calculations allowed to correlate all cluster valence electrons, except the $4 d$ electrons of the lanthanides. We used a standard IPEA value $(0.25 \mathrm{au})^{43}$ and an imaginary shift of $0.15 \mathrm{au}^{44}$

In the second step, we added the AMFI approximation of the DKH spin-orbit coupling (SOC) operator to the Hamiltonian ${ }^{45}$ and we performed restricted-active-space stateinteraction spin-orbit (RASSI-SO) ${ }^{46,47}$ calculations where all states of a given cluster computed in the first step were allowed to interact.

In these calculations, the clusters were embedded in $a b$ initio model potentials (AIMP) ${ }^{48}$ that include Coulomb, exchange, and Pauli repulsion interactions from the $\mathrm{CaF}_{2}$ host lattice obtained in Ref. 19 from self-consistent embedded-ions (SCEI) ${ }^{49}$ Hartree-Fock calculations (Ref. 27).

\section{Results}

Before we discuss the results, let us make some theoretical considerations on the onsets of the IVCT absorption bands we calculate here.

The first consideration is that the spinorbit coupling (SOC) interactions between spinorbit-free states considered in this work (cf. Table 1) would in some cases be insufficient for an accurate description of a number of lowest spinorbit states. E.g. in $\mathrm{Pr}^{3+}$, SOC interactions between the ${ }^{3} \mathrm{H}$ and ${ }^{3} F$ related states are known to be necessary. ${ }^{50}$ However, the considered SOC interactions are sufficient for the accuracies of spin-orbit ground state energies, bond lengths, and vibrational frequencies demanded for a reliable calculation of the IVCT absorptions on- sets, which are on focus here. We illustrate this with calculations of the IVCT configuration coordinate diagram and absorption band of $\mathrm{Sm}^{2+} / \mathrm{Sm}^{3+}$ pairs in $\mathrm{CaF}_{2}$, which are shown in Fig. 1.

In the left panel of Fig. 1 we show the IVCT configuration coordinate diagram as computed in this work, i.e. with SOC interactions between the ${ }^{7} F$ related states of $\mathrm{Sm}^{2+}$, on one hand, and the ${ }^{6} \mathrm{H}$ related states of $\mathrm{Sm}^{3+}$, on the other. The band profile corresponding to the first individual IVCT absorption (indicated with a vertical blue arrow on the left panel) was calculated with the parabolas of the ground state and the first IVCT state using the timedependent approach of Heller; ${ }^{51,52}$ it is shown in blue in the upper right panel. This individual IVCT absorption is a very broad band. And the same is true for all other individual IVCT absorption bands. The reason for this is the very large horizontal offset between the minima of the parabolas of the $\mathrm{Sm}^{2+}-\mathrm{Sm}^{3+}$ and $\mathrm{Sm}^{3+}-\mathrm{Sm}^{2+}$ configurations of the pair. ${ }^{18}$ This large offset is in turn a consequence of the strong relaxations that take place around the two ions of the pair after the electron transfer: after the electron leaves $\mathrm{Sm}^{2+}$, this becomes $\mathrm{Sm}^{3+}$ and the ligands get much closer to it; simultaneously, after the electron reaches $\mathrm{Sm}^{3+}$, this becomes $\mathrm{Sm}^{2+}$ and the ligands expand a lot around it. This band maximum is calculated at $14150 \mathrm{~cm}^{-1}$. Now, when the calculation is improved and SOC interactions between the ${ }^{6} H$ and ${ }^{6} F$ related states of $\mathrm{Sm}^{3+}$ are included, we get the IVCT configuration coordinate diagram of the center panel of Fig. 1. The band maximum of the first individual IVCT absorption remains practically unchanged: $14210 \mathrm{~cm}^{-1}$.

A second consideration is how the maximum of the first individual IVCT absorption is rendered in experimental spectra. For this, we recall that real IVCT absorption bands result from the convolution of many individual IVCT absorptions, due to the crowdedness of $\mathrm{Ln}^{3+}$ and $\mathrm{Ln}^{2+}$ states right above the ground state, ${ }^{3}$ which makes them broader than the individual ones. In the case of $\mathrm{Sm}^{2+} / \mathrm{Sm}^{3+}$ pairs in $\mathrm{CaF}_{2}$, the convolution of all the individual absorptions in the center panel of Fig. 1 is shown in orange 

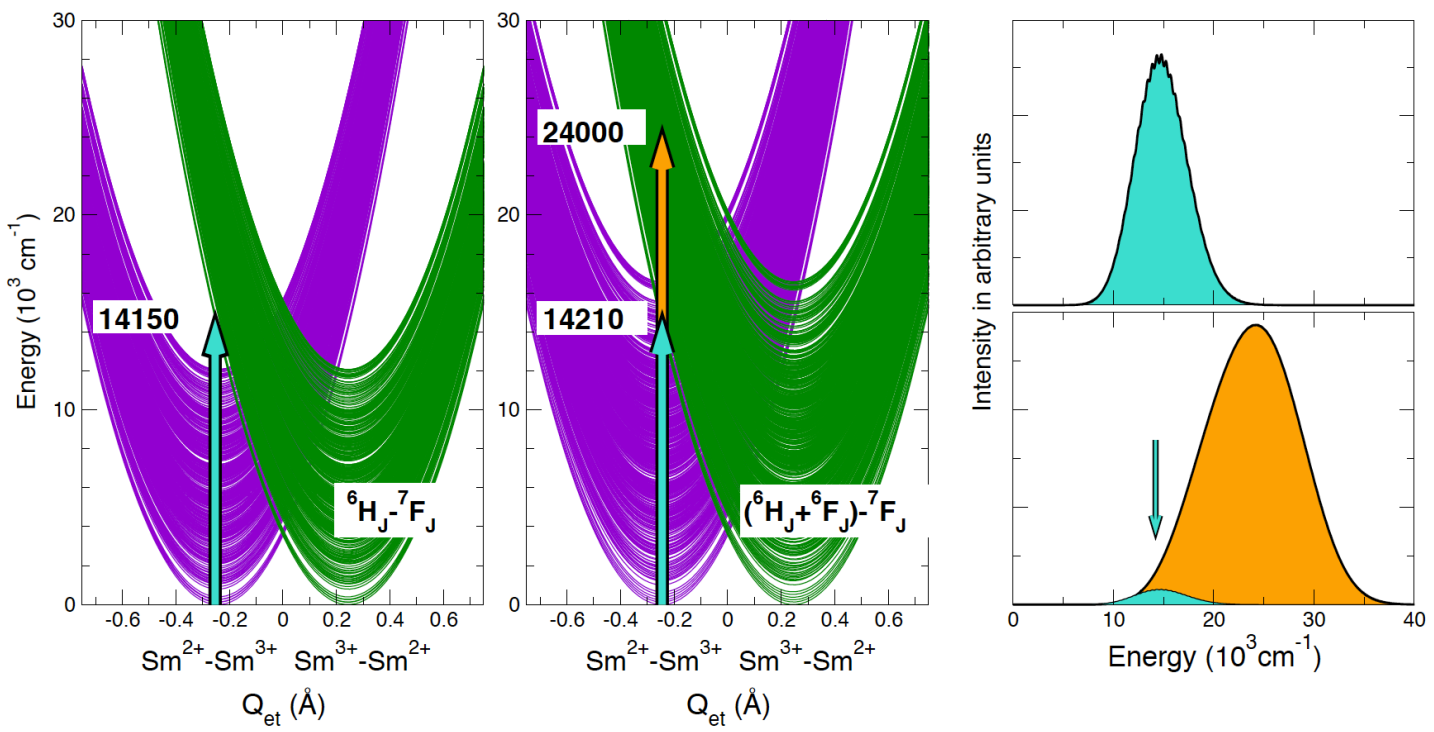

Figure 1: Left and center: IVCT energy diagrams of $\mathrm{Sm}^{2+} / \mathrm{Sm}^{3+}$ mixed valence pairs in $\mathrm{CaF}_{2}$. $\mathrm{Sm}^{2+}\left(4 f^{6}{ }^{7} F_{J}\right) / \mathrm{Sm}^{3+}\left(4 f^{5}{ }^{6} H_{J}\right)$ IVCT states (left) and $\mathrm{Sm}^{2+}\left(4 f^{6}{ }^{7} F_{J}\right) / \mathrm{Sm}^{3+}\left(4 f^{56} H_{J},{ }^{6} F_{J}\right)$ IVCT states (center). Right: first individual IVCT absorption band as calculated with the left diagram (above) and convoluted, full IVCT absorption band as calculated with the center diagram (below). The blue arrow in the right panel indicates the position of the former in the latter and illustrates how the maximum of the first individual absorption band marks the onset of the $\mathrm{Sm}^{2+} 4 f \rightarrow \mathrm{Sm}^{3+} 4 f$ IVCT absorptions. The orange arrow in the center panel indicates the energy of the full IVCT absorption maximum.

in the bottom right panel, where the contribution of the first individual IVCT absorption is shown in blue. It is clear that the maximum of the first individual IVCT absorption as calculated in this paper (upper right panel) perfectly marks the onset of the full IVCT absorption (lower right panel), as it would be obtained experimentally.

A final consideration concerns the role of states of the $4 f^{N-1} 5 d^{1}$ configuration of some divalent lanthanides, which also induce more IVCT states and hence absorptions. When their energy is higher than the $4 f^{N}$ ground state, even if they are almost degenerate, they alter the shape of the full IVCT absorption, but not its onset. When their energy is lower and the ground configuration is $4 f^{N-1} 5 d^{1}$ (this is e.g. the case of $\mathrm{Gd}^{2+}$ in $\mathrm{CaF}_{2}{ }^{23}$ ), the $4 f^{N}$ IVCT configuration coordinate diagrams calculated here do not represent the true IVCT absorptions. However, we expect the conclusions extracted here for $4 f \rightarrow 4 f$ IVCT absorption onsets also to hold in these cases, because, as we will see below, the IVCT absorption onsets are determined by the bond lengths and vibrational frequencies, and these are not very different in
$4 f^{N}$ and $4 f^{N-1} 5 d^{1}$ states. $^{53,54}$

Let us now discuss the results. In Fig. 2, we show the calculated IVCT configuration coordinate energy level diagrams of the $\mathrm{Ln}^{2+} / \mathrm{Ln}^{3+}$ pairs in $\mathrm{CaF}_{2}$ for the entire lanthanide series. In Table 2 (second column), we show the computed IVCT absorption onset of each rare earth pair (i.e. the maximum of the first individual IVCT absorption), which is also indicated in the Figure.

One can see in Fig. 2 and Tab. 2 that the $4 f \rightarrow 4 f$ IVCT absorption onset is basically constant across the series. The differences between rare earths are hardly significant having in mind the very broad nature of these absorptions. This invariance is the most remarkable feature of the results and we discuss it next.

According to the Frank-Condon rule, the maximum of the first individual IVCT absorption occurs at the energy necessary to go from $\mathrm{Ln}^{2+}-\mathrm{Ln}^{3+}$ at its equilibrium (initial state of the absorption) to $\mathrm{Ln}^{3+}-\mathrm{Ln}^{2+}$ (final state of the absorption) at fixed positions of the nuclei, i.e. without changing the atomistic structure. This energy difference is represented with vertical blue arrows in the configuration coordinate en- 

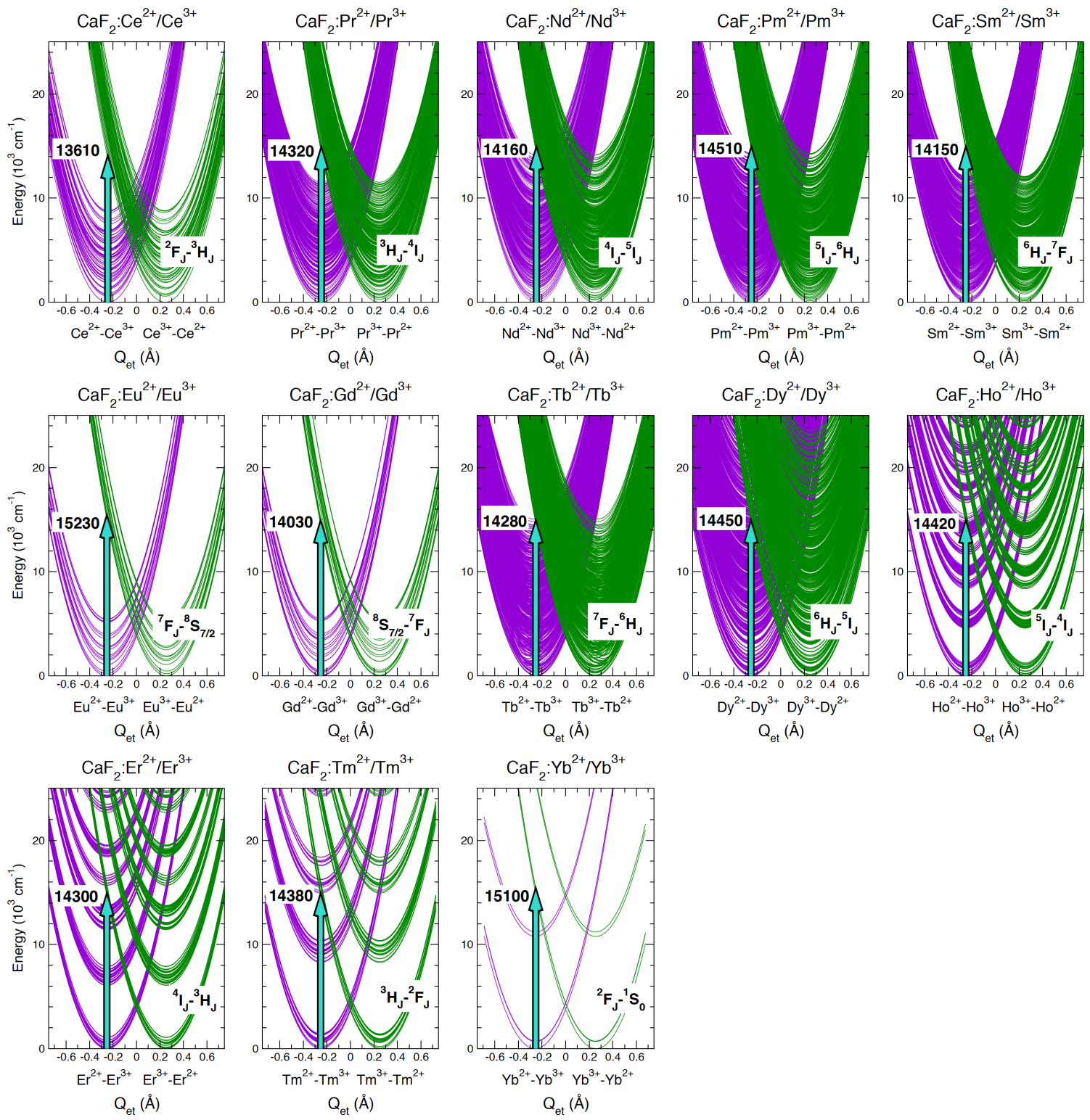

Figure 2: IVCT energy diagrams of $\mathrm{Ln}^{2+} / \mathrm{Ln}^{3+}$ mixed valence pairs in $\mathrm{CaF}_{2}$. Only $\mathrm{Ln}^{2+}\left(4 f^{N+1}\right) / \mathrm{Ln}^{3+}\left(4 f^{N}\right)$ levels are shown. The onset of the $\mathrm{Ln}^{2+} 4 f \rightarrow \mathrm{Ln}^{3+} 4 f$ IVCT absorption (understood as the maximum of the lowest individual IVCT absorption band) is indicated with a turquoise vertical arrow.

ergy diagrams of Fig. 2.

This photoinduced electron transfer is followed by no emission. Instead, a non-radiative decay with a strong structural reorganization takes place in which the ligands experience a large compression around $\mathrm{Ln}^{3+}$ and a large expansion around $\mathrm{Ln}^{2+}$. Since $\mathrm{Ln}^{2+}-\mathrm{Ln}^{3+}$ and $\mathrm{Ln}^{3+}-\mathrm{Ln}^{2+}$ have the same energies at their respective equilibrium structures, the IVCT absorption energy coincides with the structural reorganization energy, which depends entirely on structural parameters. This energy is, according to the simple predictive model of IVCT states of Ref. 26:

$$
E_{\mathrm{IVCT}}^{\mathrm{abs}}=2 k Q_{\text {et }, 0}^{2},
$$

where $k$ is an effective breathing mode force constant of donor and acceptor centers, in $\mathrm{cm}^{-1} / \AA^{2}$, and $Q_{e t, 0}$ is the electron transfer reaction coordinate, or configuration coordinate, at equilibrium, in $\AA$. They read, respectively,

$$
k=0.02966 \mu \bar{\nu}_{\text {ave }}^{2},
$$

where $\mu$ is the ligand mass (in amu) and $\bar{\nu}_{\text {ave }}$ is the average of the breathing mode vibrational 
Table 2: Computed maxima of the first $\mathbf{L n}^{2+} / \mathbf{L n}^{3+}$ IVCT absorptions (in $\mathbf{c m}^{-1}$ ), electron donor and acceptor bond lengths $(d(\mathbf{L n}-\mathbf{F})$, in $\AA)$, and breathing mode vibrational frequencies $\left(\bar{\nu}_{a_{1 q}}\right.$, in $\left.\mathbf{c m}^{-1}\right)$ of $\mathbf{C a F}_{2}: \mathbf{L n}^{2+}, \mathbf{L n}^{3+}$. Results of the absorption predicted with the IVCT model out of Donor-Aceptor bond length differences $\left(\Delta d_{D A}=d\left(\mathbf{L n}^{+2}-\mathbf{F}\right)-d\left(\mathbf{L n}^{+3} \mathbf{F}\right)\right.$, in $\left.\AA\right)$ and average vibrational frequencies $\left(\bar{\nu}_{\text {ave }}\right.$, in $\mathrm{cm}^{-1}$ ) are also given.

\begin{tabular}{|c|c|c|c|c|c|c|c|c|c|}
\hline & \multirow{2}{*}{$\begin{array}{c}\mathrm{Ln}^{2+} / \mathrm{Ln}^{3+} \\
\text { IVCT absorption } \\
\text { onset }\end{array}$} & \multicolumn{2}{|c|}{$\operatorname{Ln}^{2+}$ donor } & \multicolumn{2}{|c|}{$\mathrm{Ln}^{3+}$ acceptor } & \multicolumn{4}{|c|}{ IVCT model (Ref. 26) } \\
\hline & & $d_{\mathrm{Ln}-\mathrm{F}}$ & $\bar{\nu}_{a_{1 g}}$ & $d_{\mathrm{Ln}-\mathrm{F}}$ & $\bar{\nu}_{a_{1 g}}$ & $\Delta d_{D A}$ & $\bar{\nu}_{\text {ave }}$ & $\begin{array}{c}\text { IVCT absorption } \\
\text { onset } \\
\text { (Eq. } 4)\end{array}$ & diff. \\
\hline $\mathrm{Ce}$ & 13610 & 2.432 & 420 & 2.311 & 494 & 0.121 & 457 & 13790 & 180 \\
\hline $\operatorname{Pr}$ & 14320 & 2.422 & 426 & 2.300 & 495 & 0.122 & 461 & 14230 & -90 \\
\hline $\mathrm{Nd}$ & 14160 & 2.412 & 427 & 2.289 & 495 & 0.123 & 461 & 14500 & 340 \\
\hline $\mathrm{Pm}$ & 14510 & 2.401 & 426 & 2.278 & 495 & 0.123 & 461 & 14460 & -50 \\
\hline $\mathrm{Sm}$ & 14150 & 2.387 & 422 & 2.266 & 498 & 0.121 & 460 & 13970 & -180 \\
\hline $\mathrm{Eu}$ & 15230 & 2.387 & 421 & 2.260 & 495 & 0.127 & 458 & 15250 & 20 \\
\hline $\mathrm{Gd}$ & 14030 & 2.375 & 423 & 2.252 & 494 & 0.123 & 459 & 14340 & 310 \\
\hline $\mathrm{Tb}$ & 14280 & 2.368 & 422 & 2.244 & 494 & 0.124 & 458 & 14540 & 260 \\
\hline Dy & 14450 & 2.360 & 420 & 2.235 & 492 & 0.125 & 456 & 14650 & 200 \\
\hline Ho & 14420 & 2.352 & 420 & 2.226 & 495 & 0.126 & 458 & 14980 & 560 \\
\hline Er & 14300 & 2.345 & 419 & 2.219 & 491 & 0.126 & 455 & 14820 & 520 \\
\hline $\mathrm{Tm}$ & 14380 & 2.337 & 417 & 2.212 & 491 & 0.125 & 454 & 14520 & 140 \\
\hline $\mathrm{Yb}$ & 15100 & 2.329 & 421 & 2.200 & 491 & 0.129 & 456 & 15600 & 500 \\
\hline
\end{tabular}

frequencies of donor and acceptor centers (in $\left.\mathrm{cm}^{-1}\right)$, and

$$
Q_{e t, 0}=\sqrt{\frac{n}{2}} \Delta d_{D A},
$$

where $n$ is the coordination number of donor and acceptor, and $\Delta d_{D A}$ (in $\AA$ ) is the difference between the lanthanide-ligand distances in the donor and acceptor centers, $\Delta d_{D A}=d\left(\operatorname{Ln}^{2+}-\mathrm{L}\right)$ $-d\left(\operatorname{Ln}^{3+}-\mathrm{L}\right)$.

Eq. 1 can be rewritten in terms of $n, \mu, \bar{\nu}_{\text {ave }}$ and $\Delta d_{D A}$ as

$$
E_{\mathrm{IVCT}}^{\mathrm{abs}}=0.02966 \cdot n \cdot \mu \cdot \bar{\nu}_{\text {ave }}^{2} \cdot\left(\Delta d_{D A}\right)^{2} .
$$

We include in Table 2 the ab initio results for these structural data together with the IVCT absorption energy calculated with Eq. 4.

It is clear that the simple model correctly reproduces the $a b$ initio IVCT absorptions and their invariance across the series. Furthermore, the reliability of the model allows one to state that the reason for the invariance of the $a b i n i$ tio IVCT absorptions is the invariance of both $\bar{\nu}_{\text {ave }}$ and $\Delta d_{D A}$ across the series. The rather constant value of the vibrational frequency (and its corresponding force constant) is a manifestation of the very weak involvement of the unfilled $4 f$ shell in a bond dominated by the rare earths' $5 s, 5 p$ filled shells. The almost invariant value of $\Delta d_{D A}$ is the result of the lanthanide contraction being similar in the divalent and trivalent ions.

One should expect all these arguments to hold for other hosts and other pairs of oxidation states like $\mathrm{Ln}^{3+} / \mathrm{Ln}^{4+}$, in which case the conclusion that the IVCT absorption onset in a host is basically constant across the series would be a general conclusion. In other words, knowing (from experiments or calculations) the IVCT absorption onset of a pair of oxidations states of one rare earth in a host is sufficient to know the same property of the other rare earths in the same host.

According to this, we show in Table 3 the values of IVCT absorption energies of the lanthanides in a few hosts, as predicted by ab initio calculations. The results in CaS, $\mathrm{SrS}$, and BaS have been calculated for this work, the others are taken from literature. These data are 
Table 3: $A b$ initio IVCT absorption onsets (in $\mathrm{cm}^{-1}$ ) of mixed valence lanthanides doped in crystalline hosts.

\begin{tabular}{ccccc}
\hline Host & $\begin{array}{c}\text { Mixed } \\
\text { valence }\end{array}$ & $\begin{array}{c}\text { IVCT absorption } \\
\text { onset }\end{array}$ & $\begin{array}{c}\text { Calculated } \\
\text { Ln }\end{array}$ & Reference \\
\hline $\mathrm{CaF}_{2}$ & $\mathrm{Ln}^{2+} / \mathrm{Ln}^{3+}$ & 14500 & all & This work, 3,19 \\
$\mathrm{SrF}_{2}$ & $\mathrm{Ln}^{2+} / \mathrm{Ln}^{3+}$ & 18000 & $\mathrm{Eu,Yb}$ & 3,19 \\
$\mathrm{BaF}_{2}$ & $\mathrm{Ln}^{2+} / \mathrm{Ln}^{3+}$ & 22500 & $\mathrm{Eu}, \mathrm{Yb}$ & 3,19 \\
$\mathrm{SrCl}_{2}$ & $\mathrm{Ln}^{2+} / \mathrm{Ln}^{3+}$ & 8100 & $\mathrm{Yb}$ & 19 \\
$\mathrm{CaS}$ & $\mathrm{Ln}^{2+} / \mathrm{Ln}^{3+}$ & 10400 & $\mathrm{Eu}$ & This work \\
$\mathrm{SrS}$ & $\mathrm{Ln}^{2+} / \mathrm{Ln}^{3+}$ & 12800 & $\mathrm{Eu}$ & This work \\
$\mathrm{BaS}$ & $\mathrm{Ln}^{2+} / \mathrm{Ln}^{3+}$ & 18100 & $\mathrm{Eu}$ & This work \\
$\mathrm{SrS}$ & $\mathrm{Ln}^{3+} / \mathrm{Ln}^{4+}$ & 10000 & $\mathrm{Ce}$ & 55 (empirical) \\
$\mathrm{Cs}_{2} \mathrm{LiLuCl}_{6}$ & $\mathrm{Ln}^{3+} / \mathrm{Ln}^{4+}$ & 10000 & $\mathrm{Ce}$ & 18 \\
$\mathrm{CaTiO}$ & $\mathrm{Ln}^{3+} / \mathrm{Ln}^{4+}$ & 12000 & $\mathrm{Pr}$ & 56 \\
$\mathrm{CaZrO}_{3}$ & $\mathrm{Ln}^{3+} / \mathrm{Ln}^{4+}$ & 12800 & $\mathrm{Pr}$ & 56 \\
& & & & \\
\hline
\end{tabular}

graphically represented in Fig. 3 as a function of breathing mode force constant $k$ and electron transfer reaction coordinate at equilibrium $Q_{e t, 0}$, which can straightforwardly be obtained from Eqs. 2 and 3 using ligand masses, breathing mode vibrational frequencies, coordination numbers, and Ln-L bond length differences between donor and acceptor. The color map in the background of the figure represents the IVCT absorption onset $E_{\mathrm{IVCT}}^{\mathrm{abs}}$ according to Eq. 1 .

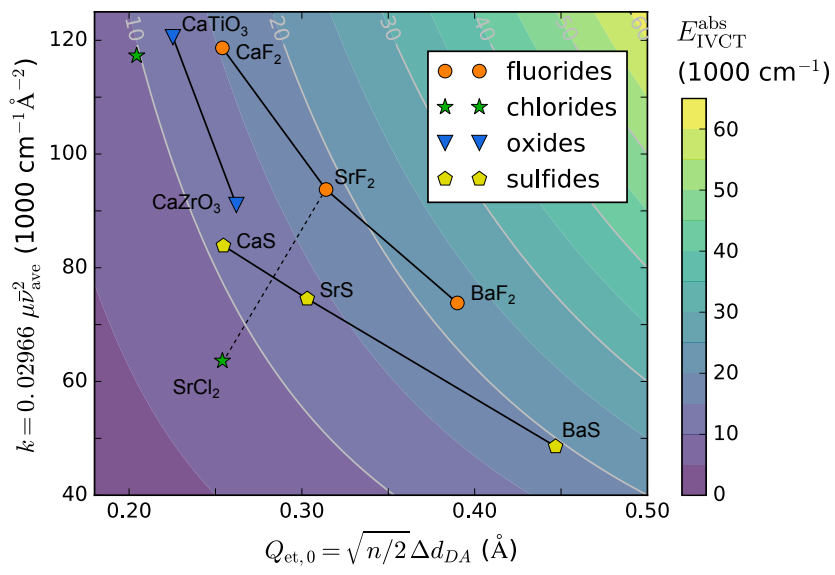

Figure 3: Calculated IVCT absorption onset $E_{\mathrm{IVCT}}^{\mathrm{abs}}$ as a function of the two parameters $Q_{e t, 0}$ and $k$ (electron transfer reaction coordinate at equilibrium and breathing mode force constant) according to Eq. 1 for a selection of host compounds. The color map and light gray isolines display the value of $E_{\mathrm{IVCT}}^{\mathrm{abs}}$. Straight lines connect isostructural hosts differing only in one cation (solid lines) or one anion (dashed line).

From this two-parameter model (Eq. 1) ${ }^{26}$ a systematic host dependence of the IVCT absorption onsets emerges that deserves further $a b$ initio study. The available data at this point indicates, nevertheless, that cation substitution, a strategy often used to fine-tune the properties of luminescent materials, impacts $E_{\mathrm{IVCT}}^{\mathrm{abs}}$ via both parameters of the model. The change in bond length upon charge transfer, $\Delta d_{D A}$, increases appreciably when going from a host with a small cation site towards one with a larger cation site, resulting in a shift to the right in the diagram of Fig. 3. Simultaneously, the vibrational frequency $\bar{\nu}_{\text {ave }}$ decreases following the bond length elongations, resulting in a downshift in Fig. 3. Both effects are visualized by solid black lines in Fig. 3 for the isostructural fluorides $\left(\mathrm{MF}_{2}, \mathrm{M}=\mathrm{Ca}, \mathrm{Sr}, \mathrm{Ba}\right)$, sulfides (MS, $\mathrm{M}=\mathrm{Ca}, \mathrm{Sr}, \mathrm{Ba})$ and perovskites $\left(\mathrm{CaAO}_{3}\right.$, $\mathrm{A}=\mathrm{Ti}, \mathrm{Zr}$ ).

Another possibility to alter the vibrational frequency is upon replacement of the anions in the compound, i.e. the ligands of the lanthanide ion. The force constant $k$, to which $E_{\text {IVCT }}^{\mathrm{abs}}$ is directly proportional, is affected because the nature of the chemical bond as well as the ligand mass will change. As an example, the dashed line in Fig. 3 illustrates the effect when moving from $\mathrm{SrF}_{2}$ to the isostructural $\mathrm{SrCl}_{2}$. This shows that replacing the anion has a roughly orthogonal effect to changing the cation in the two-dimensional parameter space. The effect on $\Delta d_{D A}$ and $k$ upon changing the ligand of the lanthanide ion results in a systematic redshift in $E_{\text {IVCT }}^{\text {abs }}$ according to the sequence $\mathrm{F}>\mathrm{O}$ $\geq \mathrm{S}>\mathrm{Cl}$. This sequence, emerging from the computed data and the model given by Eqs. 14 offers a semi-quantitative tool to predict the location of IVCT states for mixed valence lanthanide pairs, $\mathrm{Ln}^{2+} / \mathrm{Ln}^{3+}$ or $\mathrm{Ln}^{3+} / \mathrm{Ln}^{4+}$ in a variety of host compounds.

Since IVCT states can cause luminescence quenching and even complete luminescence excitation loss, ${ }^{3}$ according to the present IVCT diagrams (cf. Fig. 2), it is suggested that the IVCT absorption onsets reported are in fact emission energy thresholds, i.e., all emissions of higher energies than them are expected to be partly or totally quenched by the presence of the respective mixed valence pairs. E.g., the presence of $\mathrm{Ln}^{2+} / \mathrm{Ln}^{3+}$ mixed valence pairs in $\mathrm{CaF}_{2}$ is expected to partly or totally quench all emissions of an energy higher than $14000 \mathrm{~cm}^{-1}$. In some cases, like $\mathrm{Tb}$ and Dy, the crowded- 
ness of states suggests that all emissions well above that threshold will be quenched. In other cases, like $\mathrm{Tm}$ and $\mathrm{Yb}$, the discontinuity of states creates energy windows where metastable states and their emissions can exist, although the mixed valence pairs are expected to partly quench them, leading to a worsened thermal quenching behavior and a lowered photoluminescence quantum efficiency. Finding these metastable states above the first IVCT absorption energies demands careful analysis case by case.

\section{Conclusions}

Multiconfigurational ab initio calculations on divalent/trivalent mixed-valence lanthanide ions doped in $\mathrm{CaF}_{2}$ show that the IVCT absorption onset is basically invariant across the lanthanide series in a given host. The onset sets an energy threshold for partial or total luminescence quenching when mixed-valence pairs are formed in the lanthanide-doped host.

The invariance of the IVCT absorption onset is rationalized by using a simple model of IVCT absorptions. ${ }^{26}$ According to it, the IVCT invariance is a consequence of respective invariances of the breathing mode vibrational frequency, on one hand, and the difference between lanthanide-ligand bond lengths in the two oxidation states, $d\left(\mathrm{Ln}^{2+}-\mathrm{L}\right)-d\left(\mathrm{Ln}^{3+}-\mathrm{L}\right)$, on the other. The latter is, in turn, a consequence of the lanthanide contractions of divalent and trivalent ions being very similar. The model points out systematic host dependences of the lanthanide IVCT absorption onsets.

Acknowledgement This work was partially supported by Ministerio de Economía y Competitividad, Spain (Dirección General de Investigación y Gestión del Plan Nacional de $\mathrm{I}+\mathrm{D}+\mathrm{i}$, MAT2017-83553-P).

\section{References}

(1) Verhoeven, J. W. Glossary of Terms Used in Photochemistry (IUPAC Recommenda- tions 1996). Pure Appl. Chem. 1996, 68, 2223-2286.

(2) Nic, M.; Jirat, J.; Kosata, B. IUPAC Gold Book. https://doi.org/10.1351/ goldbook. I03125, 2006-; Accessed: 201906-10.

(3) Joos, J.; Seijo, L.; Barandiarán, Z. Direct Evidence of Intervalence Charge-Transfer States of Eu-Doped Luminescent Materials. J. Phys. Chem. Lett. 2019, 10, 15811586 .

(4) Marcus, R. A. Chemical and Electrochemical Electron-Transfer Theory. Annu. Rev. Phys. Chem 1964, 15, 155-196.

(5) Allen, G. C.; Hush, N. S. IntervalenceTransfer Absorption. Part 1. Qualitative Evidence for Intervalence-Transfer Absorption in Inorganic Systems in Solution and in the Solid State. Prog. Inorg. Chem. 1967, 8, 357-389.

(6) Robin, M.; Day, P. Mixed Valence Chemistry - A Survey and Classification. Adv. Inorg. Chem. Radiochem. 1968, 10, 247422.

(7) Berkooz, O.; Malamud, M.; Shtrikman, S. Observation of Electron Hopping in ${ }^{151} \mathrm{Eu}_{3} \mathrm{Eu}_{4}$ by Mössbauer Spectroscopy. ssc 1968, 6, 185-188.

(8) Wickleder, C. A New Mixed Valent Europium Chloride: $\mathrm{Na}_{5} \mathrm{Eu}_{7} \mathrm{Cl}_{22}$. Z. Naturforsch. 2002, 57b, 901-907.

(9) Wickleder, C. $\mathrm{KEu}_{2} \mathrm{Cl}_{6}$ and $\mathrm{K}_{1.6} \mathrm{Eu}_{1.4} \mathrm{Cl}_{5}$ : Two New Mixed-Valent Europium Chlorides. Z. Anorg. Allg. Chem. 2002, 628, 1815-1820.

(10) Shulg'in, B. V.; Buzmakova, S. I.; Viktorov, L. V.; Krymov, A. L.; Petrov, V. L.; Podurovskii, S. V.; Kozlov, A. A.; Shapiro, B. M.; Shrom, M. Y.; Nepomnyashchikh, A. I. et al. Scintillation Detectors Working with $\mathrm{CaF}_{2}$-Eu SingleCrystals. Atom. Energy 1993, 75, 534538. 
(11) Leblans, P.; Vandenbroucke, D.; Willems, P. Storage Phosphors for Medical Imaging. Materials 2011, 4, 1034-1086.

(12) Smet, P. F.; Parmentier, A. B.; Poelman, D. Selecting Conversion Phosphors for White Light-Emitting Diodes. J. Electrochem. Soc. 2011, 158, R37-R54.

(13) Abé, S.; Joos, J. J.; Martin, L. I. D. J.; Hens, Z.; Smet, P. F. Hybrid Remote Quantum Dot/Powder Phosphor Designs for Display Backlights. Light: Sci. Appl. 2017, 6, e16271.

(14) Maldiney, T.; Bessiere, A.; Seguin, J.; Teston, E.; Sharma, S. K.; Viana, B.; Bos, A. J. J.; Dorenbos, P.; Bessodes, M.; Gourier, D. et al. The In Vivo Activation of Persistent Nanophosphors for Optical Imaging of Vascularization, Tumours and Grafted Cells. Nature Materials 2014, 13, 418-426.

(15) Botterman, J.; Smet, P. F. Persistent Phosphor $\mathrm{SrAl}_{2} \mathrm{O}_{4}$ :Eu,Dy in Outdoor Conditions: Saved by the Trap Distribution. Opt. Express 2015, 23, A868-A881.

(16) Xu, J.; Tanabe, S. Persistent Luminescence Instead of Phosphorescence: History, Mechanism, and Perspective. J. Lumin. 2019, 205, 581-620.

(17) van Schaik, W.; Lizzo, S.; Smit, W.; Blasse, G. Influence of Impurities on the Luminescence Quantum Efficiency of (La,Ce,Tb) $\mathrm{PO}_{4}$. J. Electrochem. Soc. 1993, 140, 216-222.

(18) Seijo, L.; Barandiarán, Z. Intervalence Charge Transfer Luminescence: The Anomalous Luminescence of Ce-Doped $\mathrm{Cs}_{2} \mathrm{LiLuCl}_{6}$ Elpasolite. J. Chem. Phys. 2014, 141, 214706/1-214706/14.

(19) Barandiarán, Z.; Seijo, L. Intervalence Charge Transfer Luminescence: Interpaly Between Anomalous and $5 d-4 f$ Emissions in Yb-Doped Florite-Type Crystals. J. Chem. Phys. 2014, 141, 234704.
(20) Strek, W.; Tomala, R.; Marciniak, L.; Lukaszewicz, M.; Cichy, B.; Stefanski, M.; Hreniak, D.; Kedziorski, A.; Krosnicki, M.; Seijo, L. Broadband anti-Stokes white emission of $\mathrm{Sr}_{2} \mathrm{CeO}_{4}$ nanocrystals induced by laser irradiation. Phys. Chem. Chem. Phys. 2016, 18, 27921-27927.

(21) MacKeen, C.; Bridges, F.; Kozina, M.; Mehta, A.; Reid, M. F.; Wells, J.P. R.; Barandiarán, Z. Evidence That the Anomalous Emission from $\mathrm{CaF}_{2}: \mathrm{Yb}^{2+}$ Is Not Described by the Impurity Trapped Exciton Model. J. Phys. Chem. Lett. 2017, 8, 3313-3316.

(22) MacKeen, C.; Bridges, F.; Seijo, L.; Barandiarán, Z.; Kozina, M.; Mehta, A.; Reid, M. F.; Wells, J.-P. R. The Complexity of the $\mathrm{CaF}_{2}: \mathrm{Yb}$ System: A Huge, Reversible, X-ray-Induced Valence Reduction. J. Phys. Chem. C 2017, 121, 2843528442 .

(23) McClure, D. S.; Kiss, Z. Survey of the Spectra of the Divalent Rare-Earth Ions in Cubic Crystals. J. Chem. Phys. 1963, 39, 3251 .

(24) Merz, J. L.; Pershan, P. S. Charge Conversion of Irradiated Rare-Earth Ions in Calcium Fluoride. I. Phys. Rev. 1967, 162, 217-235.

(25) Merz, J. L.; Pershan, P. S. Charge Conversion of Irradiated Rare-Earth Ions in Calcium Fluoride. II. Thermoluminescent Spectra. Phys. Rev. 1967, 162, 235-247.

(26) Barandiarán, Z.; Meijerink, A.; Seijo, L. Configuration Coordinate Energy Level Diagrams of Intervalence and Metal-toMetal Charge Transfer States of Dopant Pairs in Solids. Phys. Chem. Chem. Phys. 2015, 17, 19874-19884.

(27) Karlström, G.; Lindh, R.; Malmqvist, P. A.; Roos, B. O.; Ryde, U.; Veryazov, V.; Widmark, P. O.; Cossi, M.; Schimmelpfennig, B.; Neogrady, P. et al. 
MOLCAS: A Program Package for Computational Chemistry. Comput. Mater. Sci. 2003, 28, 222-239.

(28) Douglas, M.; Kroll, N. M. Quantum Electrodynamical Corrections to the Fine Structure of Helium. Ann. Phys. (N.Y.) 1974, 82, 89-155.

(29) Hess, B. A. Relativistic ElectronicStructure Calculations Employing a Two-Component No-Pair Formalism with External-Field Projection Operators. Phys. Rev. A 1986, 33, 3742-3748.

(30) B. O. Roos, R. Lindh, P. A. Malmqvist, V. Veryazov, P. O. Widmark,; Borin, A. C. New Relativistic Atomic Natural Orbital Basis Sets for Lanthanide Atoms with Applications to the Ce Diatom and $\mathrm{LuF}_{3}$. J. Phys. Chem. A 2008, 112, 11431-11435.

(31) Roos, B. O.; Lindh, R.; Malmqvist, P. A.; Veryazov, V.; Widmark, P. O. Main Group Atoms and Dimers Studied with a New Relativistic ANO Basis Set. J. Phys. Chem. A 2004, 108, 2851-2858.

(32) Pascual, J. L.; Seijo, L.; Barandiarán, Z. $\mathrm{Ab}$ initio model potential study of environmental effects on the Jahn-Teller parameters of $\mathrm{Cu}^{2+}$ and $\mathrm{Ag}^{2+}$ impurities in $\mathrm{MgO}, \mathrm{CaO}$ and $\mathrm{SrO}$ hosts. J. Chem. Phys. 1993, 98, 9715 .

(33) Roos, B. O.; Taylor, P. R.; Siegbahn, P. E. M. A Complete Active Space SCF Method (CASSCF) Using a DensityMatrix Formulated Super-CI Approach. Chem. Phys. 1980, 48, 157-173.

(34) Siegbahn, P. E. M.; Heiberg, A.; Roos, B. O.; Levy, B. Comparison of the Super-CI and the Newton-Raphson Scheme in the Complete Active Space SCF Method. Phys. Scr. 1980, 21, 323-327.

(35) Siegbahn, P. E. M.; Heiberg, A.; Almlöf, J.; Roos, B. O. The Complete Active Space SCF (CASSCF) Method in a
Newton-Raphson Formulation with Application to the HNO Molecule. J. Chem. Phys. 1981, 74, 2384-2396.

(36) Froese-Fischer, C. Oscillator Strengths for ${ }^{2} S-{ }^{2} P$ Transitions in the Copper Sequence. J. Phys. B 1977, 10, 1241.

(37) T. H. Dunning, Jr., B. H. Botch,; J. F. Harrison, On the orbital description of the $4 \mathrm{~s} 3 \mathrm{~d}(\mathrm{n}+1)$ states of the transition metal atoms. J. Chem. Phys. 1980, 72, 3419.

(38) Barandiarán, Z.; Seijo, L. Radial correlation effects on interconfigurational excitations at the end of the lanthanide series: A restricted active space secons order perturbation study of $\mathrm{Yb}^{2+}$ and $\mathrm{SrCl}_{2}: \mathrm{Yb}^{2+}$. J. Chem. Phys. 2013, 138, 074102.

(39) Andersson, K.; Malmqvist, P.-A.; Roos, B. O.; Sadlej, A. J.; Wolinski, K. Second-Order Perturbation Theory with a CASSCF Reference Function. J. Phys. Chem. 1990, 94, 5483-5488.

(40) Andersson, K.; Malmqvist, P.-A.; Roos, B. O. Second-Order Perturbation Theory with a Complete Active Space Self-Consistent Field Reference Function. J. Chem. Phys. 1992, 96, 1218-1226.

(41) Zaitsevskii, A.; Malrieu, J.-P. MultiPartitioning Quasidegenerate Perturbation Theory. A New Approach to Multireference Møller-Plesset Perturbation Theory. Chem. Phys. Lett. 1995, 233, 597604 .

(42) Finley, J.; Malmqvist, P.-A.; Roos, B. O.; Serrano-Andrés, L. The Multi-State CASPT2 Method. Chem. Phys. Lett. 1998, 288, 299-306.

(43) G. Ghigo, B. O. Roos,,; P.-A. Malmqvist, A modified definition of the zeroth-order Hamiltonian in multiconfigurational perturbation theory (CASPT2). Chem. Phys. Lett. 2004, 396, 142. 
(44) Forsberg, N.; Malmqvist, P.-A. Multiconfiguration Perturbation Theory with Imaginary Level Shift. Chem. Phys. Lett. 1997, 274, 196.

(45) Hess, B. A.; Marian, C. M.; Wahlgren, U.; Gropen, O. A Mean-Field Spin-Orbit Method Applicable to Correlated Wavefunctions. Chem. Phys. Lett. 1996, 251, 365-371.

(46) Malmqvist, P. A.; Roos, B. O.; Schimmelpfennig, B. The RASSI Approach with Spin-Orbit Coupling. Chem. Phys. Lett. 2002, 357, 230-240.

(47) Paulovic, J.; Nakajima, T.; Hirao, K.; Lindh, R.; Malmqvist, P.-A. Relativistic and Correlated Calculations on the Ground and Excited States of ThO. J. Chem. Phys. 2003, 119, 798-805.

(48) Barandiarán, Z.; Seijo, L. The Ab Initio Model Potential Representation of the Crystalline Environment. Theoretical Study of the Local Distortion on NaCl: $\mathrm{Cu}^{+}$. J. Chem. Phys. 1988, 89, 5739-5746.

(49) Seijo, L.; Barandiarán, Z. Ab Initio Model Potential Study of Local Distortions Around $\mathrm{Cr}^{+}$and $\mathrm{Cr}^{3+}$. J. Chem. Phys. 1991, 94, 8158.

(50) Krośnicki, M.; K? D? Ydziorski, A.; Seijo, L.; Barandiarán, Z. Ab Initio Theoretical Study on the $4 f^{2}$ and $4 f 5 d$ Electronic Manifolds of Cubic Defects in $\mathrm{CaF}_{2}: \mathrm{Pr}^{3+}$. J. Phys. Chem. A 2014, 118, 358-68.

(51) Heller, E. J. Time-Dependent Approach to Semiclassical Dynamics. J. Chem. Phys. 1975, 62, 1544.

(52) Heller, E. J. The Semiclassical Way to Molecular Spectroscopy. Acc. Chem. Res. 1981, 14, 368.

(53) Barandiarán, Z.; Seijo, L. In Computational Methods in Lanthanide and Actinide Chemistry; Dolg, M., Ed.; John Wiley \& Sons: New York, 2015; pp 217-240.
(54) de Jong, M.; Meijerink, A.; Seijo, L.; Barandiarán, Z. Energy Level Structure and Multiple $4 f^{12} 5 d^{1}$ Emission Bands of $\mathrm{Tm}^{2+}$ in Halide Perovskites: Theory and Experiment. J. Phys. Chem. C 2017, 121, 10095-10101.

(55) Kulesza, D.; Cybińska, J.; Seijo, L.; Barandiarán, Z.; Zych, E. Anomalous Red and Infrared Luminescence of $\mathrm{Ce}^{3+}$ Ions in SrS:Ce Sintered Ceramics. J. Phys. Chem. C 2015, 119, 27649-27656.

(56) Barandiarán, Z.; Bettinelli, M.; Seijo, L. Color Control of $\mathrm{Pr}^{3+}$ Luminescence by Electron-Hole Recombination Energy Transfer in $\mathrm{CaTiO}_{3}$ and $\mathrm{CaZrO}_{3}$. J. Phys. Chem. Lett. 2017, 8, 3095-3100. 